\title{
Parsing with C++ Deferred Expressions
}

\author{
Damian Conway \\ Victorian Centre for Image Processing and Graphics \\ Department of Computer Science \\ Monash University \\ damian@bruce.cs.monash.edu.au
}

\begin{abstract}
A technique for constructing embedded grammar-based parsers in $\mathrm{C}++$ was described in [1]. This paper presents an extension to that technique which allows parsing actions to be specified directly as part of the grammar, rather than indirectly (as function calls).
\end{abstract}

\section{Introduction}

The Parser class library ${ }^{1}$ defines a set of related classes which may be used to build parsing grammars within a $\mathrm{C}++$ program. Extensive use of operator overloading enables these grammars to be specified with a syntax very similar to the familiar yacc format. The library includes features such as lazy evaluation of terminals, user-defined resolution of shift-reduce and reduce-reduce conflicts, polymorphic value assignments to terminals and non-terminals, context-sensitive parsing, and the embedding of actions within a parser.

The library as presented in [1] has one major limitation - the specification of actions to be performed during parsing is restricted to embedded pointers to functions. When a parsing rule is successfully matched, these function pointers (suitably encapsulated in Action class objects) are dereferenced and their corresponding functions called.

Because function definitions in $\mathrm{C}++$ cannot be nested, the use of function pointers to specify parser actions necessarily implies that the code corresponding to the actions must be physically separate from the grammar in which those actions are used. Although it produces clean and modular code, this style of building parsers is somewhat tedious. More importantly, the resultant embedded grammars are considerably more difficult to understand than the equivalent yacc specification.

This paper introduces an additional set of classes which implement a C-like interpreted language suitable for specifying parser actions. Statements in the language are converted to an expression tree encapsulated in a class object. Such "expression" objects can then be included as part of an embedded grammar.

\section{The Deferred Expression Idiom}

Van Wyk [2] describes a technique for building a set of classes which encode and solve simultaneous equations specified in a declarative manner within a $\mathrm{C}++$ program. The technique overloads common operations such as addition, subtraction and equality test, causing such operators not to perform the corresponding operation, but to generate an object which represents the operation. Such representative objects can then be passed to a suitable modified constraint solver [3] in which the operations they represent may be evaluated at need.

In a similar manner, the classes described in this paper used overloaded operators (in this case, virtually all of the operators in the $\mathrm{C}++$ language) to generate an object representing the parse tree of the expression. This expression object belongs to a class derived from the root $\mathrm{Parser}$ class, and hence the original expressions used to generate it may be incorporated seamlessly into any embedded grammar built with the Parser library. Figure 1 illustrates the technique, components of which are described in Sections 3 through 8.

During parsing, expression objects (like the Action objects they supersede) are matched trivially against the input stream. That is, as part of a rule to be matched, expression objects are ignored and therefore consume none of the input stream.

Once a rule has been successfully matched, each of its components are evaluated by calling their virtual Evaluate () method, which must be added to the Parser base class. In order to initiate this match-evaluate sequence, a new method, Parse (), is added to the Rule class. This method attempts to match the grammar

\footnotetext{
${ }^{1}$ As described in [1] and available by anonymous ftp from bruce.cs.monash.edu. au in the directory

/pub/damian/Parser.shar.z.uu
} 
rooted at its parent Rule against the input stream and, if successful, evaluates the resultant parser tree and returns its final value.

Terminals and rules within the grammar evaluate trivially (that is, their Evaluate () methods do nothing) whilst objects of class Action call the function associated with them. Calling the Evaluate () method of one of the new expression objects causes the entire expression tree contained in it to be evaluated, effectively executing the original expression embedded in the grammar.

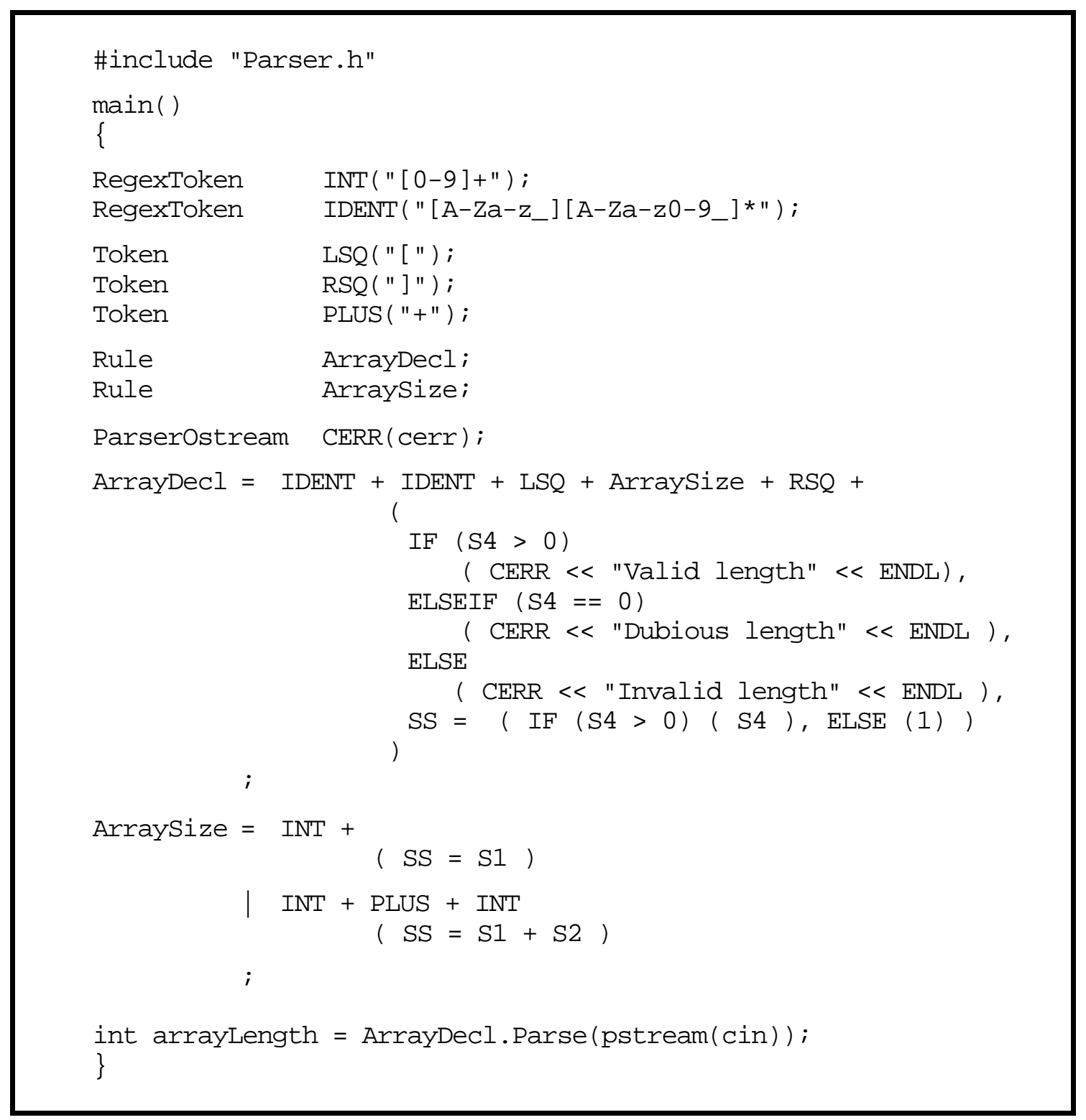

Figure 1: Use of deferred expressions in an embedded parser..

\section{The ParserExpr Base Class}

In order to simplify the construction and evaluation of expression trees, all classes representing deferred expressions are derived from a common base class, ParserExpr, which is in turn directly derived from the root class Parser. Hence ParserExpr objects can be placed in embedded parser specifications, just like Token, Rule and Action objects.

The ParserExpr class extends class Parser by adding two protected members, ths and rhs, of type ParserExpr*. These members are used to implement the (predominantly binary) expression tree, although some derived classes add additional members to handle $\mathrm{N}$-ary expressions such as cascaded ifs (see Section 8). The ParserExpr class provides a constructor which initializes its two members (to zero, by default). The constructor is protected, ensuring that the base class itself is never instantiated. 
Classes corresponding to useful operations can then be derived from the ParserExpr class, by redefining their virtual Evaluate () methods. This method computes and returns the value of the expression rooted at the current object. See Sections 5 through 8 for examples. Note that the virtual Match () method of each derived class can be inherited from the ParserExpr base class without redefinition, because ParserExpr: :Match () is defined to match any input stream trivially (that is, simply to return the value of the object's this pointer.)

Instances of derived expression classes are created within a grammar by defining the appropriate overloaded operators (again, see Sections 5 through 8 for examples).

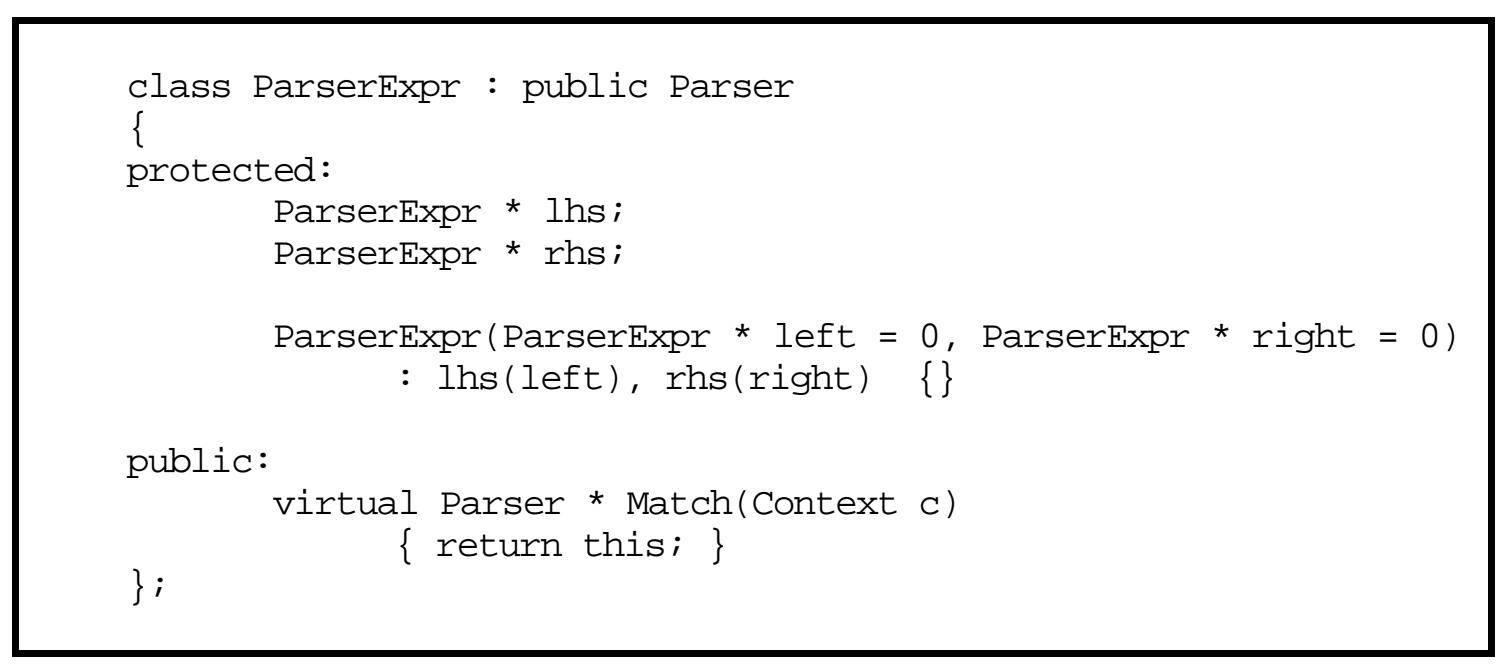

Figure 2: A base class for representing deferred expressions.

\section{Embedding References To Grammar Components}

The basis of most useful parser actions is the manipulation of values matched by individual terminals or rules during the parse. To this end, parser specification languages typically provide a mechanism for referring to the current value of individual components of a rule. In the yacc specification language, for example, these references are known as $\$ 1, \$ 2$, etc., and correspond to the values of first, second, etc. components of a right hand side of a rule. The left hand side of a rule can also be referred to, as $\$ \$$.

To create such references within the deferred expressions described in this paper, a special class, ParserVar, is derived from class ParserExpr. A ParserVar object contains an integer member, index, indicating the ordinal position within the current parent rule of the component being referred to. When the Evaluate () method of such an object is called, it interrogates the Context object passed as its argument and returns the value of the appropriate component of the parent rule. This is equivalent to evaluating $\$$ index in a yacc specification. Figure 3 illustrates the necessary class definition.

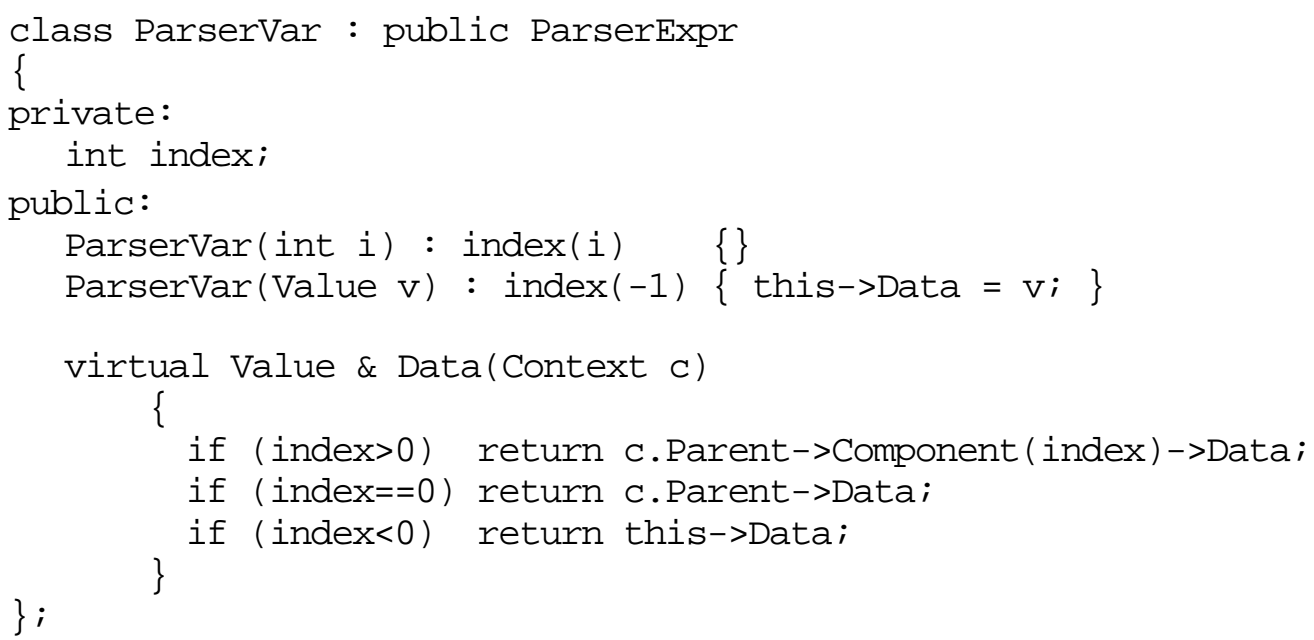

Figure 3: A class for representing grammar component values. 
Two values of the member index are reserved for special purposes. If index is zero, the ParserVar refers to the value of the parent rule of the current context, and hence is equivalent to $\$$ \$ in a yacc specification. If index is negative, the ParserVar object represents a literal value, which is stored in the Data member (inherited from class Parser) of the object. The capacity to store values literally (as well as by reference) is critical, as it permits expressions containing literal values to be embedded in a grammar. Class ParserVar provides a special constructor, which takes a literal value as its single argument, stores it in its Data member, and automatically sets index to -1 .

It is convenient to predefine certain frequently used instances of class ParserVar in order to simplify the construction of appropriate grammars. As the use of the dollar sign as the first character of an identifier is not permitted in the proposed ISO/ANSI C++ standard, in the Parser class library the character 'S' has been used to replace it, yielding SS for $\$ \$, S 1$ for $\$ 1, \mathrm{~S} 2$ for $\$ 2$, etc. Figure 1 illustrates the use of these globals in deferred expressions.

\section{Embedding Deferred Assignments}

Experience indicates that the single most common expression embedded in a Parser grammar is the simple assignment, such as: $S S=S 1$ (which assigns the value of the first component of a rule to the rule itself; effectively passing a value back up the parse tree.)

This deferred assignment must be carried out when the rule has been successfully matched and is being evaluated. Hence the assignment operator of class ParserVar is overloaded as shown in Figure 2. Note the overloading for an argument of type Value. This enables literal values to be used as the assigned value in a deferred assignment, once they have been suitably encapsulated in a ParserVar object.

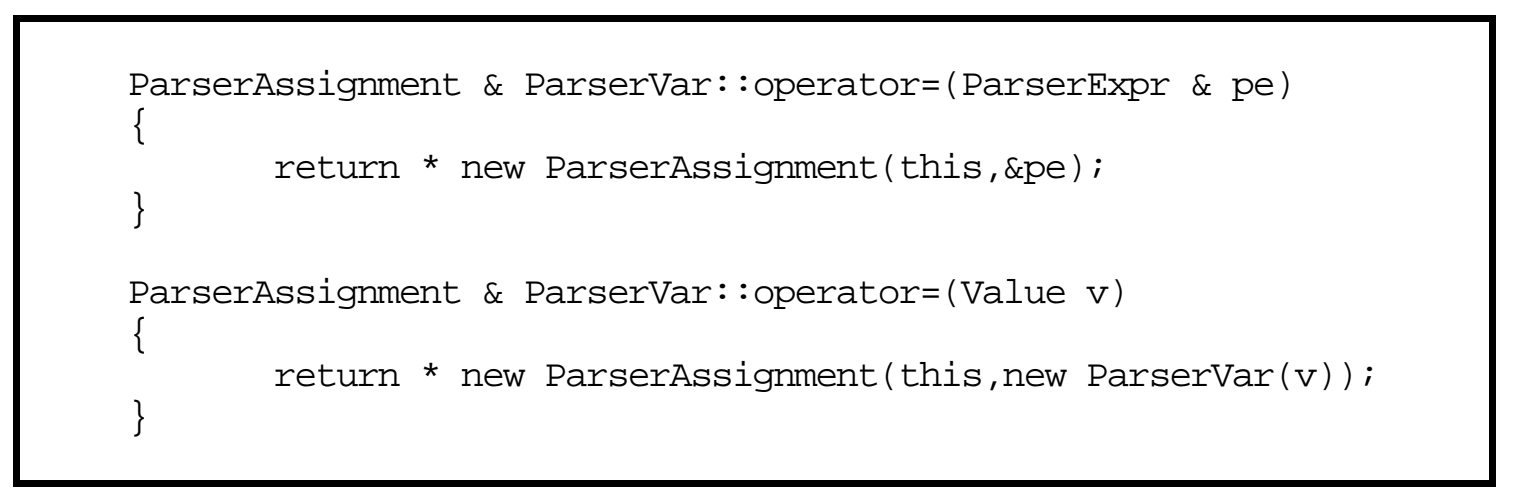

Figure 4: A deferred assignment operator.

The ParserAssignment class is derived from ParserExpr and stores pointers to a ParserVar (the target of the assignment) and a ParserExpr (representing the value to be assigned) in its 1 hs and rhs members. When a ParserAssignment object is evaluated, it executes the deferred assignment, as indicated in Figure 4. As is standard in $\mathrm{C}$ and $\mathrm{C}++$, the evaluation of the assignment returns the assigned value, so that deferred assignments may be chained or otherwise embedded in larger expressions.

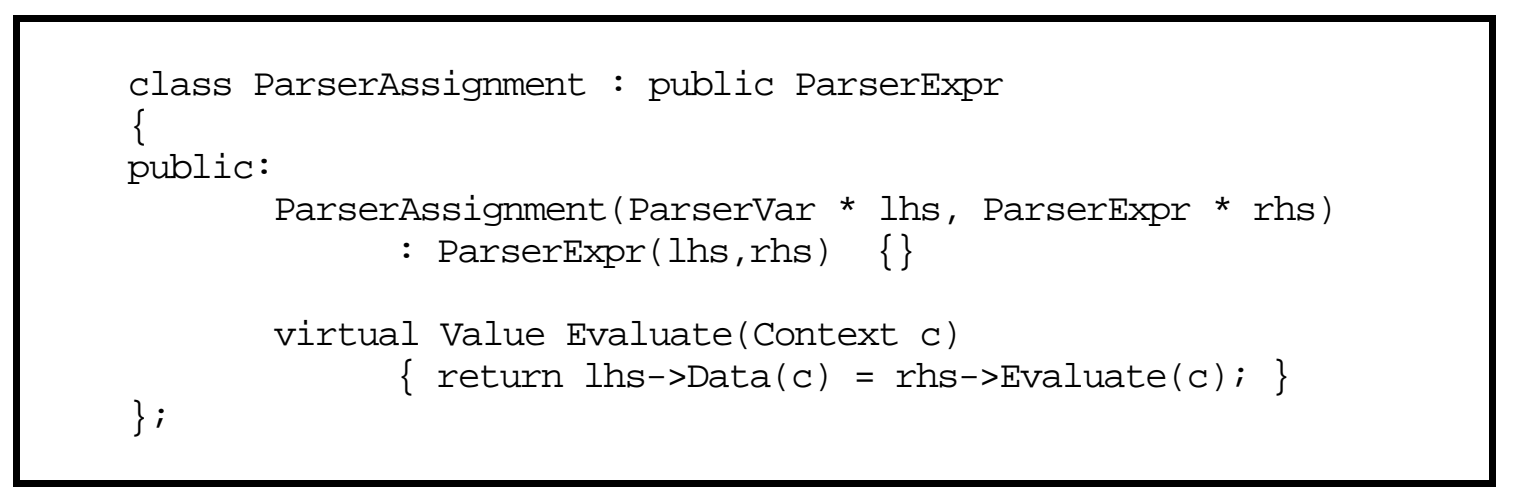

Figure 5: A class for representing deferred assignments.

Similar classes representing other deferred assignment operations can easily be created by adding the corresponding overloaded operator to the ParserVar class, creating the corresponding deferred assignment class, and redefining the Evaluate () method of the new class to perform the appropriate operation. 


\section{Embedding Other Binary Operations}

The same approach can be used to create other types of deferred binary operators by creating suitable expression classes (for example, deferred addition as presented in Figure 6.) Note the structural and functional similarity between the ParserAddition and ParserAssignment classes. In fact, the only difference between the two is that ParserAddition objects are created using a global overloaded operator (since such operations are not required to be class methods, as is the overloaded assignment operation.) Again notice the two versions of the operator with literal arguments.

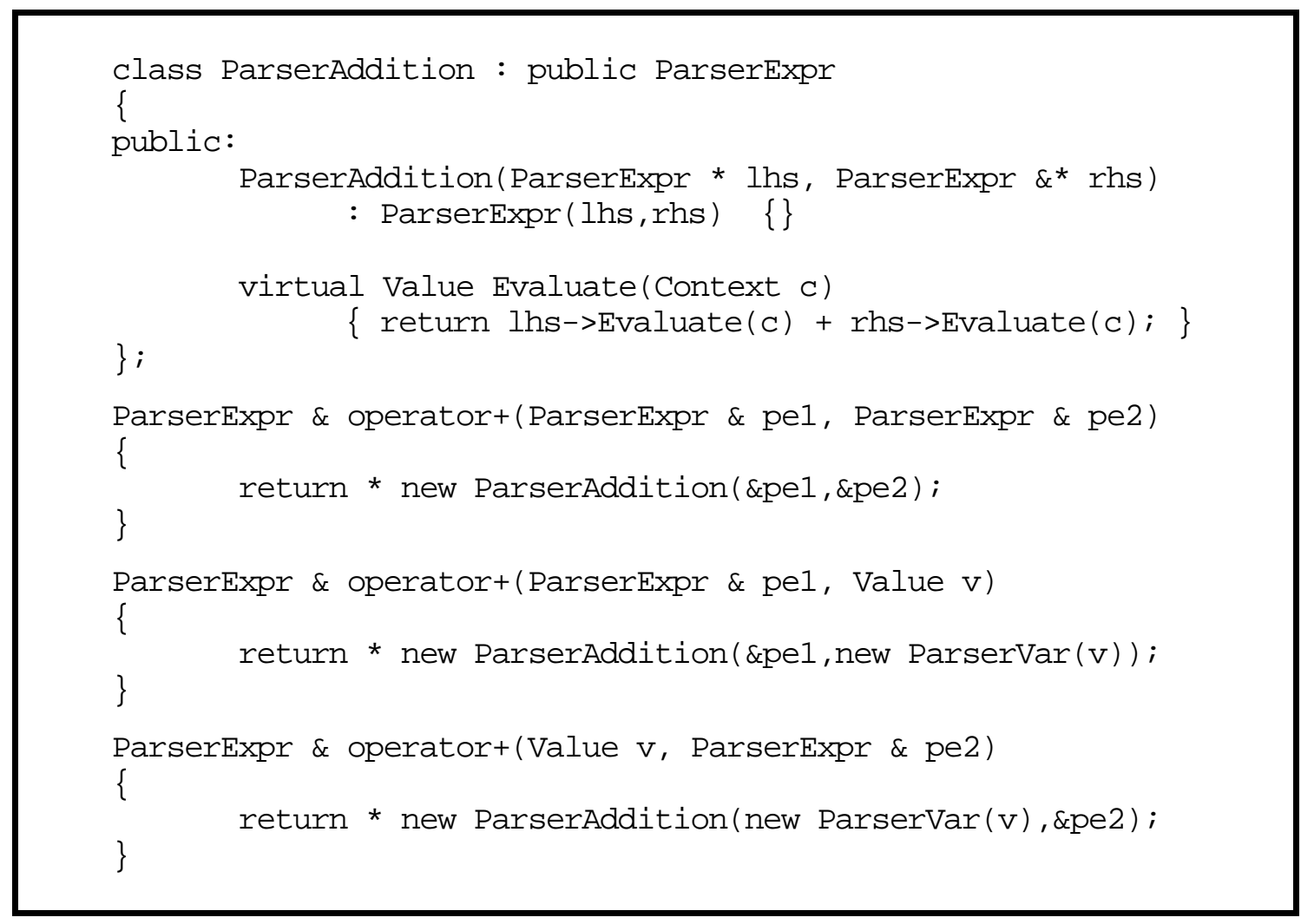

Figure 6: A class for representing deferred additions.

\section{Embedding Deferred Output}

Yet another common action during parsing is output. In $\mathrm{C}++, \mathrm{I} / \mathrm{O}$ is handled via overloaded binary operators. The paradigm is easily reproduced for deferred expressions by creating two new classes (see Figure 7). This section presents an implementation of deferred output (the more common operation required during a parse) but the technique is readily adapted to implement deferred input as well.

The first additional class required is ParserOstream, which is used to encapsulate ostream objects ${ }^{1}$ using a private reference. Note that ParserOstream need not be derived from ParserExpr. However doing so allows a ParserOstream object to be used as a test condition in a deferred selection construct (see Section 8 below).

The second necessary class, ParserOutput, is used to represent complete deferred output operations and must be derived from class ParserExpr. A ParserOutput object stores a reference to an ostream and a list of pointers to ParserExpr objects, whose values are to later to be evaluated and written to the ostream.

A Parseroutput object is created when the overloaded left shift operator (operator $<<$ ) of a ParserOstream object is called with an argument of type ParserExpr. An overloaded left shift operator is also added as a method of class ParserOutput, to permit chaining of output operations (as demonstrated in

\footnotetext{
${ }^{1}$ In fact, this class is not strictly necessary, as the global left shift operator could be overloaded for arguments of type ostream\& and ParserExpr\&. However, such an approach would preclude the use of literal values as the second argument in a deferred output operation, since operator $<<$ (ostream\&, Value\&) is already overloaded to implement undeferred output of Value objects.
} 
Figure 1). Each left shift operation causes a pointer to the second argument (a ParserExpr) to be added to the list in the ParserOutput object. The completed ParserOutput object is then included in a particular Rule, which is subsequently parsed. As with all other deferred expressions the ParserOutput object trivially matches during the parse phase and then performs the required deferred output when its Evaluate () method is called.

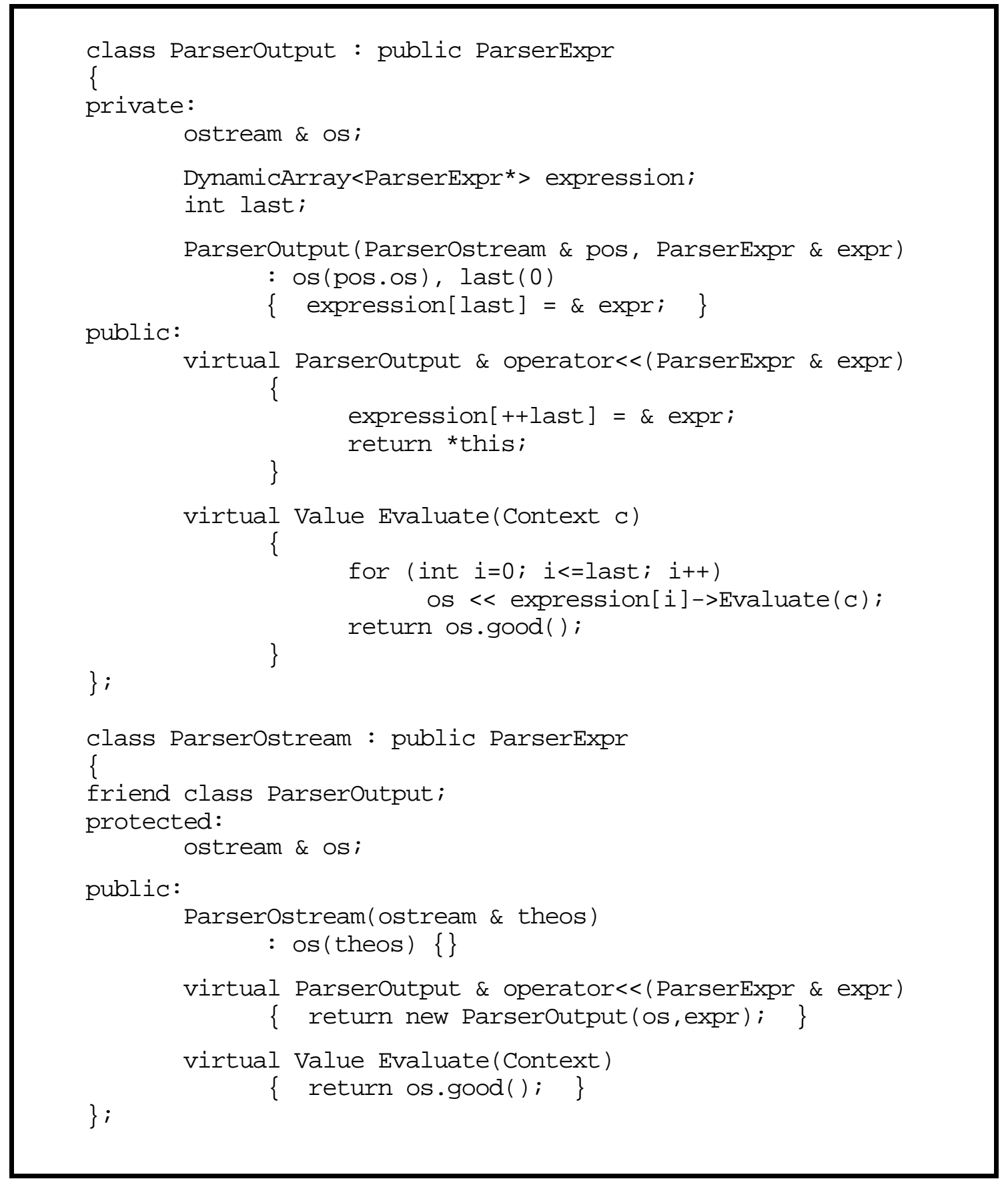

Figure 7: Classes to support deferred output.

\section{Embedding Control Structures}

One important binary operation that must treated slightly differently is the sequencing operation: operator, (). By itself, there is no reason that sequencing of expressions could not be accomplished simply by defining a global operator, () function that returns a ParserSequence object. However, it is also desirable to provide conditional sequencing - a deferred if statement - and this requires somewhat more sophistication from the comma operator. 
Instead of being declared as a global function, the comma operator is defined as a virtual method of class ParserExpr itself (as in Figure 8). In addition, another overloaded sequence operator is defined, taking a second argument of class ParserAlternative. Objects of this class represent the alternative actions in a (cascaded) if construct. This additional comma operator simply produces an error message in the general case, but will be redefined to do something more useful in the Parser If class presented below.

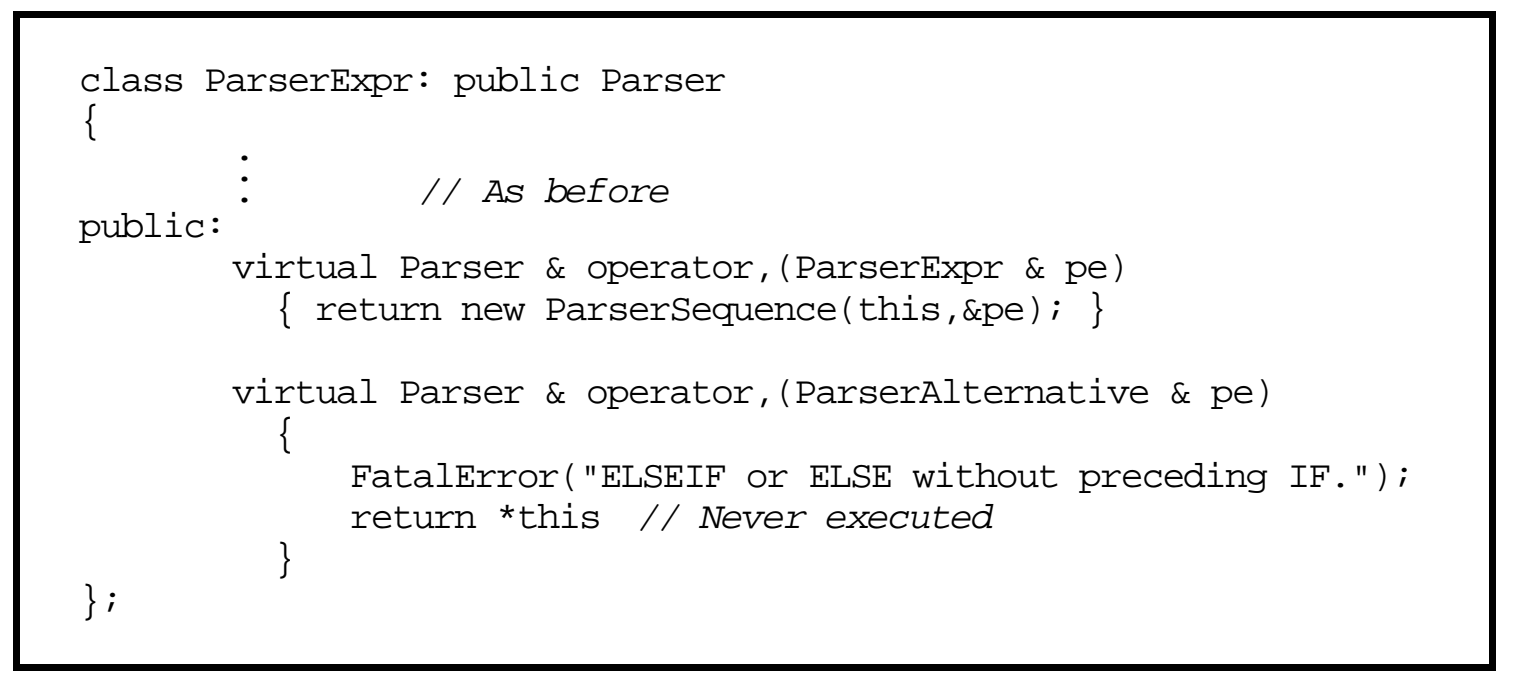

Figure 8: The virtual sequence operator for ParserExpr.

In order to permit the use of selection statements within deferred expressions, a special class, ParserIf, is created. Unlike most of its sibling classes, ParserIf contains two dynamic arrays of ParserExpr pointers, enabling it to store a series of alternate conditions and actions. A helper class, ParserAlternative, is also required, but need not belong to the ParserExpr hierarchy. Figure 9 illustrates the necessary code.

Selection constructs are created using the global IF () function. This creates a ParserIf object and initializes its first condition to point to the function argument. The expression to be returned (if the deferred condition evaluates to non-zero) is then passed to the ParserIf object using the function call operator. This two step approach produces a syntax very similar to the standard $C / C++$ syntax (see Figure 1), except that brackets are used to contain the body of the deferred if, rather than braces.

Another significant difference is that a ParserIf object represents an expression rather than a statement, hence its result may be used in subsequent expressions (see Figure 1 for an example). Thus the deferred if also provides the functionality of the ternary operator (?:). Indeed, this particular situation is one in which seems to have been overlooked ${ }^{1}$ by Stroustrup and Ellis in their prohibition [4] of an overloaded operator?: () .

Class ParserIf redefines the virtual sequence operator it inherits from class ParserExpr, so that any ParserAlternative object which follows a deferred if expression are appended to the list of possible condition/expression pairs.

ParserAlternative objects are created through the global function ELSEIF (), and contain pointers to a deferred condition and a corresponding deferred expression ${ }^{2}$. These pointers are appended to the respective lists in the preceding ParserIf. ParserAlternative objects may also be created using the global function ELSE (), in which case their condition pointer is set to zero, which the method ParserIf: : Evaluate () subsequently interprets as "always succeed".

\section{Conclusion}

The techniques presented in this paper greatly improve the usability of the Parser classes presented in [1]. The approach illustrated can be extended to any binary or unary $\mathrm{C}++$ operator which can be overloaded. Other extensions which might be considered include support for deferred function calls, other control structures (in particular, loops), and support for a dynamic record type (as an extension of the value class).

\footnotetext{
${ }^{1}$ This is not intended as a criticism: this application of operator overloading is quite esoteric and was hardly likely to suggest itself to the hardworking language designers.

${ }^{2}$ Class ParserAlternative is structurally and functionally very similar to class ParserIf. Objects of class ParserAlternative are creating with a one argument constructor which stores a pointer to the deferred condition, and completed using their own operator ( ) to store a pointer to the corresponding deferred expression.
} 
The concept of deferred expressions also has wide application beyond this particular problem domain. Applications in the areas of lazy evaluation, extensible interpreters, and declarative or self-modifying styles of programming will readily suggest themselves to the reader.

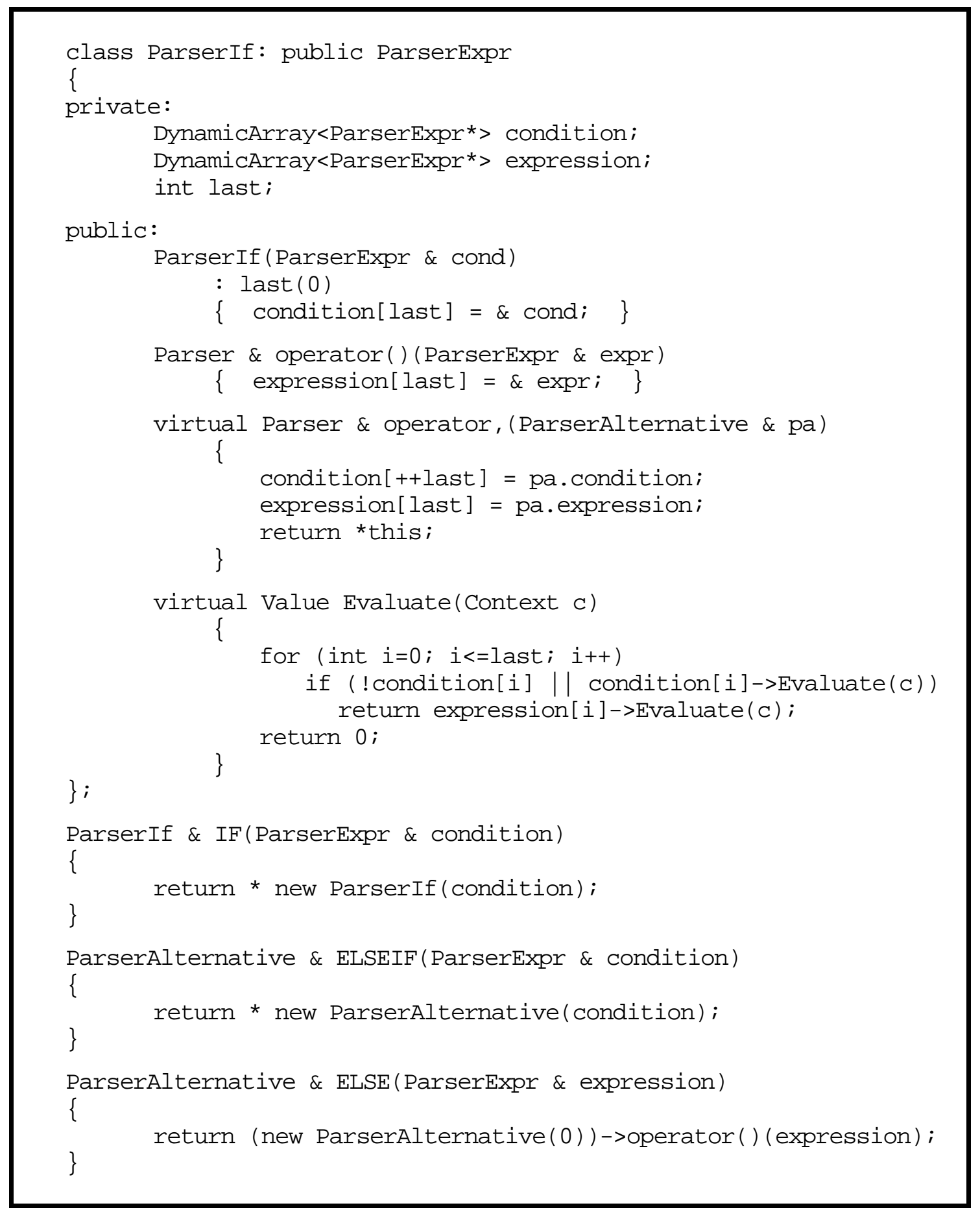

Figure 9: Code to support deferred selection constructs.

\section{References}

[1] Conway, D.M., Parsing with C++ Classes, ACM SIGPLAN Notices, vol. 29, no. 1, pp. 46-52, January 1994.

[2] Van Wyk, C.J., Arithmetic Equality Constraints as C++ Statements, Software - Practice and Experience, vol. 22, no. 6, pp. 467-494, 1992.

[3] Derman, E. \& Van Wyk, C.J., A Simple Equation Solver and its Application to Financial Modelling, Software Practice and Experience, vol. 14, no. 12, pp. 1169-1184, 1984.

[4] Ellis, M, \& Stroustrup, B., The Annotated C++ Reference Manual, §13.4 , Addison-Wesley, 1992. 